\title{
Visual Composition of Complex Queries on an Integrative Genomic and Proteomic Data Warehouse
}

\author{
Francesco Pessina, Marco Masseroli, Arif Canakoglu \\ Dipartimento di Elettronica e Informazione, Politecnico di Milano, Milan, Italy \\ Email: francesco.pessina87@gmail.com,marco.masseroli@polimi.it, canakoglu@elet.polimi.it
}

Received December 2012

\begin{abstract}
Biomedical questions are usually complex and regard several different life science aspects. Numerous valuable and heterogeneous data are increasingly available to answer such questions. Yet, they are dispersedly stored and difficult to be queried comprehensively. We created a Genomic and Proteomic Data Warehouse (GPDW) that integrates data provided by some of the main bioinformatics databases. It adopts a modular integrated data schema and several metadata to describe the integrated data, their sources and their location in the GPDW. Here, we present the Web application that we developed to enable any user to easily compose queries, although complex, on all data integrated in the GPDW. It is publicly available at http://www.bioinformatics.dei.polimi.it/GPKB/. Through a visual interface, the user is only required to select the types of data to be included in the query and the conditions on their values to be retrieved. Then, the Web application leverages the metadata and modular schema of the GPDW to automatically compose an efficient SQL query, run it on the GPDW and show the extracted requested data, enriched with links to external data sources. Performed tests demonstrated efficiency and usability of the developed Web application, and showed its and GPDW relevance in supporting answering biomedical questions, also difficult.
\end{abstract}

Keywords: SQL Query Composition; Visual Interface; Integrated Data Extraction; Data Warehousing; Bioinformatics Database

\section{Introduction}

A great amount of valuable and heterogeneous biomedical molecular data and information is increasingly produced thanks to the modern high-throughput technologies. It is stored in publicly accessible molecular biology databases that are continuously increasing in number and coverage of the included biomolecular entities, as well as of their described structural and functional biomedical features and associated phenotypes [1]. Such databases provide extremely valuable information to infer new knowledge and potentially answer biomedical questions, which are generally complex. To obtain enough evidence to support such answers, several different structural, functional and phenotypic annotations must be collected and comprehensively evaluated. Yet, even the information about a single biomolecular entity is often scattered across many different sources.

Several approaches have been proposed to integrate data from multiple heterogeneous data sources and query them comprehensively. Data warehousing well supports off-line processing to mine the integrated data towards knowledge discovery. Yet, the global schemas for biological data warehousing that have been proposed are quite complex [2-5]. Although they can support a complete representation of the underlying biological aspects described by the integrated data, they make it difficult to face the integration challenges of evolving data. Difficulties arise in both maintaining updated the data warehouse that adopts one of such data schemas and in expanding it with other data and data types from new sources.

To overcome such difficulties, we created a Genomic and Proteomic Data Warehouse (GPDW) that adopts an original metadata-based modular global schema [6]. It supports integration of data sources that evolve in data content, structure (although limitedly) and number, as the biomedical molecular ones do. Thanks to the adopted data schema, we easily kept updated and extended progressively the numerous genomic, proteomic and phenotypic controlled annotation data of different species integrated in the GPDW. Here, we describe and discuss the original Web application that we developed to easily access and search such valuable integrated biomolecular knowledge. It leverages the GPDW metadata-based modular data schema to enable any user to visually perform queries, although complex, whose extracted data can support answering difficult biomedical questions. 


\section{Genomic and Proteomic Data Warehouse}

\subsection{Integrative Data Schema}

To integrate heterogeneous data available from many different sources, abstraction and generalization of concepts to be integrated are paramount. As well, modularity and customizability of global data schema are vital to support easy integration, data schema extension with the inclusion of new data types and sources, and maintenance with respect to data, format and schema evolution of the integrated original data sources. With such aims and with the goal of creating the GPDW, as illustrated in [6], we focused on biomedical molecular entities and features described by data to be integrated, provided by distinct sources. Briefly, we abstracted and generalized such features and defined our integrated relational data schema as composed of multiple interconnected modules. Each module represents a single feature, whose data are provided by one or more of the integrated data sources, and it is composed by a number of data tables that depends on the integrated data. Such tables are hierarchically related as shown in the Directed Acyclic Graph (DAG) in Figure 1.

Feature modules can be pair wise associated; such associations represent the valuable association/annotation data provided by the integrated data sources, which are stored in hierarchically related association tables (Figure 2).

The feature modules and their associations contained in a specific instance/version of our generalized global data schema depend on the particular data sources and their provided data that are integrated in that specific data schema instance. To support the automatic construction

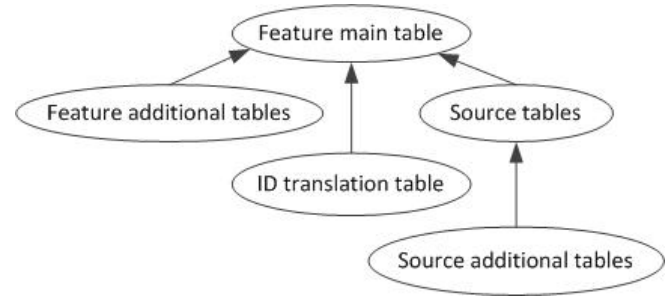

Figure 1. Directed acyclic graph of the tables in a GPDW feature module.

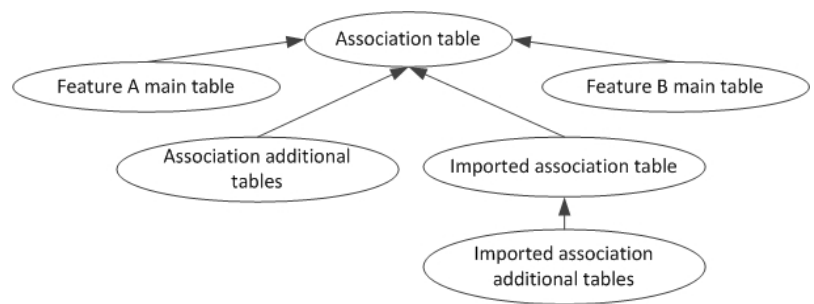

Figure 2. DAG of the association tables between two GPKB feature modules. and updating of a database adopting such data schema, we defined a procedure to register the data sources and their feature data to be integrated, and to collect all the required metadata information about them and their associations. We store these metadata in a specific metadata schema, useful to seamlessly and transparently access all data in the database regardless the specific database version.

\subsection{Data Integrated in the GPDW}

The GPDW adopts our defined modular data schema to integrate data provided by several of the main bioinformatics databases, including Entrez Gene, Homologene, MINT, IntAct, Expasy Enzyme, GO, GOA, BioCyc, KEGG, Reactome, eVOC and OMIM. Currently, data in the GPDW regard several features, including DNA sequences, genes, transcripts, proteins, enzymes, protein domains, small molecules of biological interest, biological function features (i.e. Gene Ontology biological processes, molecular functions and cellular components), pathways, gene expression features, genetic disorders, clinical synopses and their association.

Among others, at time of writing the GPDW contains 9,537,645 genes of 9,631 organisms, 38,960,202 proteins of 338,004 species, 19,522 protein domains and 824,797 protein domains annotations, 28,889 biochemical pathways and 171,372 pathway annotations (77,812 gene and 93,560 protein annotations), 35,252 Gene Ontology terms and 64,185,070 Gene Ontology annotations (1,272,168 gene and 62,912,902 protein annotations), 10,212 human genetic disorders and their 27,705 gene annotations. These figures demonstrate the valuable unique characteristics of the GPDW.

\section{Dynamic Composition and Result Visualization of GPDW Data Extraction SQL Queries}

To enable any user to easily compose queries, although complex, on all data integrated in the GPDW, we developed a Web application in Java programming language using Servlets and Java Server Pages (JSP) technology. It is publicly available at

http://www.bioinformatics.dei.polimi.it/GPKB/. Through a visual interface (Figure 3), the user is only required to select, out of the features integrated in the GPDW, the ones and their attributes to be included in the query, together with the conditions on the data values to be retrieved. All information about the GPDW content required to build the visual interface is taken from the GPDW metadata. Thus, transparently to the user, the visualized features and their attributes automatically adapt to the content of the specific GPDW instance.

Interactive menus, present in the visual interface for 


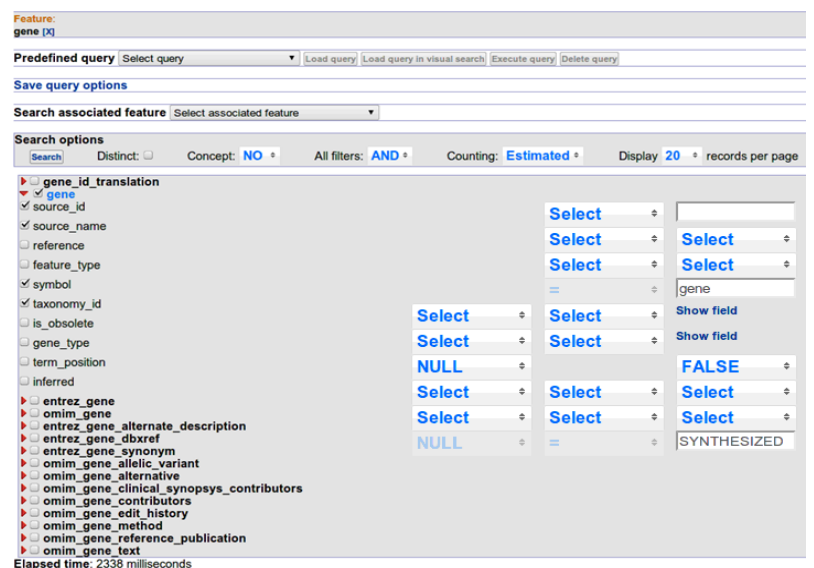

Figure 3. User interface for visual composition of a query on a single feature.

each feature attribute, enable the user to define filtering conditions on the data values to be retrieved. The content of each of these menus depends on the data contained in the particular attribute to which the menu refers. Furthermore, the user can also select some general options (Search Options) for the query, e.g. make a "distinct" SQL query, set the conjunctive logical clause to use for the defined filtering conditions (AND/OR), select the result counting mode and define how many results per page to show.

\subsection{Query Composition Algorithm}

We designed a specific algorithm to automatically compose a SQL query that can efficiently extract data from any instance of the GPDW, according to any combination of user selected feature attributes and filters. The core of the query composition algorithm leverages the metadata and modular structure of the GPDW. It is divided in two steps: 1) generation of a complete and ordered list of tables to be included in the query and 2) generation of the query FROM, JOIN and WHERE clauses.

The first step starts with the identification of the lowest common ancestor (LCA) between all the tables that contain the attributes selected by the user to be included in the query. This is performed as shown in Figure 4, according to the DAG structure in Figures $\mathbf{1}$ and 2. Through a recursive function, all the ancestors of each table are calculated and the LCA table between all of them is found. It completes the list of all tables involved in the query, which also includes the tables that contain the attributes selected by the user. Then, the tables in the list are ordered based on the feature module, or feature association they belong to and the order in which the user selected the features to query. If the user selected first the feature $\mathrm{A}$ and then the feature $\mathrm{B}$, the feature A tables will be at the top of the list, then the association tables be-

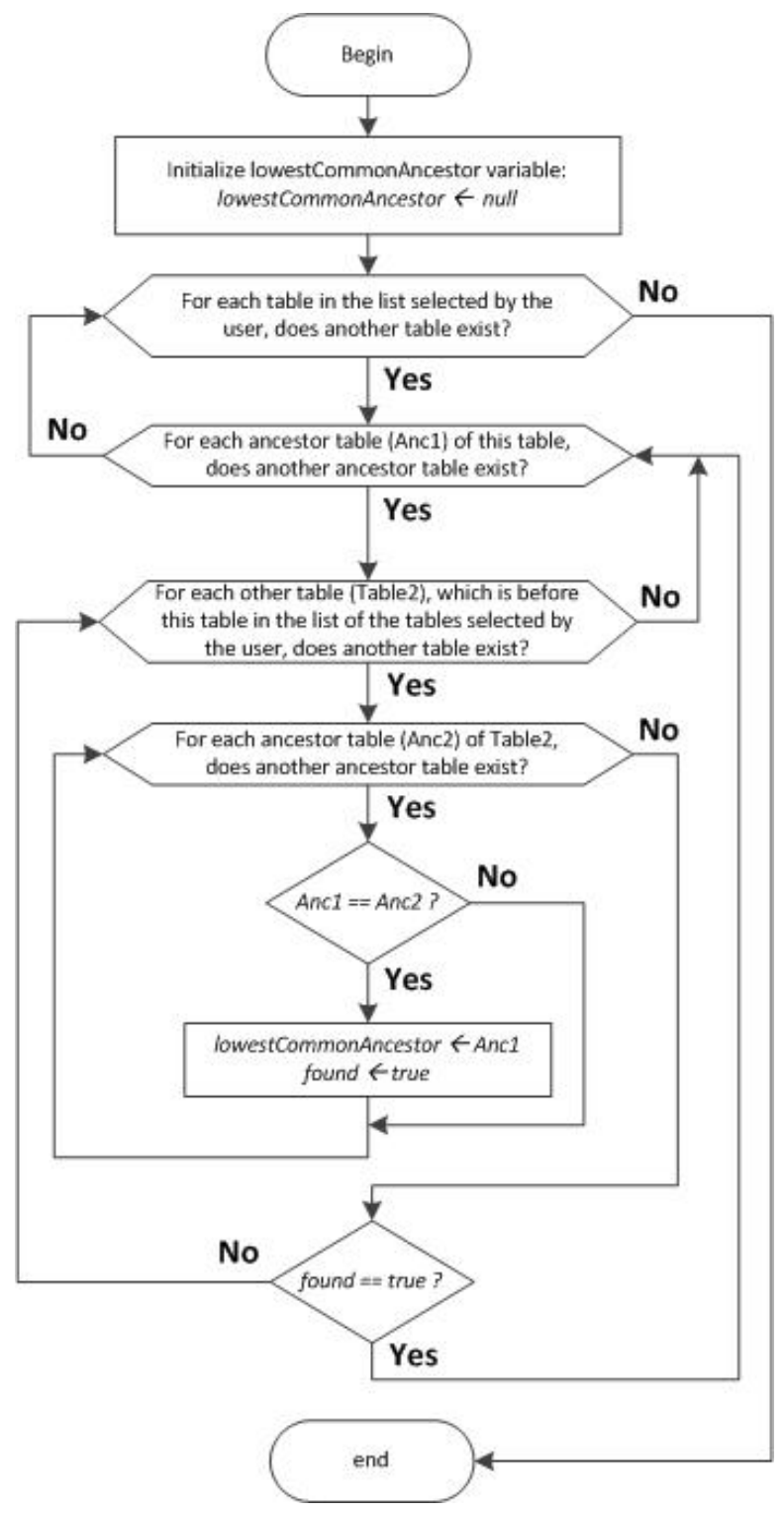

Figure 4. Flowchart of the lowest common ancestor search over the list of all ancestor tables of each GPDW table containing user selected attributes.

tween feature A and feature B, and finally the feature B tables. In the list, the order of tables belonging to each feature module or association is then defined from top to bottom by the order of tables in the DAG of the feature module (Figure 1) or of the association (Figure 2).

In the second step, the query join generation is performed as follows. The first table of the list is the table inserted into the FROM clause of the query and set as reference table for the joins with the following tables in the list. Then, the ordered table list is scanned from top to bottom and each table in the list is evaluated. During this scan, when a feature or association table is found, it becomes the reference table for the joins with the following tables in the list. If it is a feature table, no join is added 
into the query. If it is an association table, the two joins between this association table and each of the two feature tables (if present in the list) that it associates are added into the query. If the table is neither a feature nor an association table, the join between this table and the last reference table found is added into the query. Finally, all filters defined by the user are added to the WHERE clause of the query.

\subsection{Query Result Visualization}

The data extracted by the composed query are processed to be neatly visualized to the user. Furthermore, ID data are enriched with hyperlinks to external resources with more detailed information over the entity identified by the ID. Each of these hyperlinks is composed of the ID itself and a base URL stored in the GPDW metadata.

Counting of the extracted data is also provided. Since the counting query is generally much slower than the main data extraction query, initially an approximate count is shown to the user. It is obtained by the SQL EXPLAIN command, quickly executed over the main query, while the real counting query is executed in background. When this query ends, in the user interface the approximate count is refreshed with the exact one.

\section{System Performance}

Performance of the created Web application was tested while running on a TomCat 6.0 Web Server installed on a computer with two Intel XEON CPU E5320 1.86 GHz, 32 GB RAM, and connected to the described GPDW instance implemented in a PostgreSQL DBMS. Generation of the visual user interface for query composition, execution of the composed queries and query result visualization processing were evaluated.

\subsection{Visual User Interface for Query Composition}

The loading times of the visual interface for query composition on a single feature are shown in Table 1. The "Servlet Execution Times" are those of a servlet created to retrieve from the GPDW metadata schema all the table names of the feature selected by the user and to create a Web page where showing them. The "JavaScript Execution Times" refer to the tasks performed by some created AJAX calls to the server or client-side JavaScript routines. This JavaScript processing shows the table field names in the user interface and populates their interactive menus for the definition of the query filtering conditions. In the default visualization, only the fields of the feature main table (and association table(s) in case of a search on more features) are immediately shown. The other table fields can be interactively opened by the user. This pena-
Table 1. Loading times of the visual user interface for single feature query composition.

\begin{tabular}{lccc}
\hline Feature & $\begin{array}{c}\text { Servlet } \\
\text { Execution } \\
\text { Time (ms) }\end{array}$ & $\begin{array}{c}\text { JavaScript } \\
\text { Generation } \\
\text { Time }(\mathrm{ms})\end{array}$ & $\begin{array}{c}\text { Total Time } \\
(\mathrm{ms})\end{array}$ \\
\hline Biological Function Feature & 517 & 416 & 933 \\
Clinical Synopsis & 155 & 423 & 578 \\
DNA Sequence & 65 & 324 & 389 \\
Enzyme & 134 & 351 & 485 \\
Gene & 334 & 400 & 734 \\
Gene Expression Feature & 405 & 352 & 757 \\
Genetic Disorder & 226 & 491 & 717 \\
Pathway & 114 & 558 & 672 \\
Protein & 97 & 324 & 421 \\
Protein Family \& Domain & 60 & 589 & 649 \\
Small Molecule & 69 & 294 & 363 \\
Transcript & 58 & 329 & 387 \\
Mean & 186.17 & 404.25 & 590.42 \\
Standard Deviation & 153.74 & 96.23 & 182.52 \\
\hline & & &
\end{tabular}

lizes slightly the total loading time of the query composition Web page for a single feature with few tables. Yet, it makes the total loading time of the query composition Web page much lower in the case of more features, which involves many tables. The total loading times are always lower than 1 second, which represents an immediate response to the user according to [7], and show a very good performance.

\subsection{Query Execution Performance}

Execution performance of the composed queries was tested on a single, two and multiple features. All queries got a low execution time, also in the cases where several tables were involved and millions of tuples were found. For example, a query over the "gene" and "biological function feature" associated features, which involved 3 tables, found 10,647,473 tuples in an execution time of $753 \mathrm{~ms}$. Besides to the good GPDW data structure and indexing, this performance is also due to the created query composition algorithm, which generates queries with an optimized number of joins. By taking advantage of the modular structure of the GPDW, in the generated query the algorithm tends not to include all the tables and joins which would be required by following the foreign key path among the tables that contain all user selected feature attributes. Instead, it only includes the minimum number of tables and joins needed to return the same results. In so doing, the algorithm generates efficient and neat queries. 


\subsection{Result Processing and Visualization}

Processing times to visualize enriched the results returned by a query on the "transcript" feature, which selects all, or only the default, feature attributes, are reported in Table 2. As expected, the processing time is proportional to the number of attributes requested and result rows visualized. For all attributes, until 300 rows, the processing time stays under 10 seconds, which is an acceptable loading time according to [7] if a feedback to the user is shown (as it is). For more rows the time is greater, but still under a minute. In any case, the benefit of an enriched visualization of result data is much more worthy than the time the user has to wait to see the results.

\section{Usability Testing}

Usability of the created Web application was tested by 9 users with 3 different profiles: 4 computer scientists who knew the GPDW, 4 computer scientists who did not know before the GPDW and 1 biologist. All users were observed while doing some significant tasks over the Web application and were interviewed about their user experience. In the evaluation, for each task, the metrics used were the success rate, the time, the number of undo/errors and the time perceived by each user. All users completed all tasks, some with assistance (success rate $80 \%$ ). This is a strong indication that the Web application does not present any critical issue. The number of undo/errors was very low (in few tasks the users did

Table 2. Visualization processing time of data extracted with a query on the "transcript" feature.

\begin{tabular}{ccc}
\hline Visualized Rows & $\begin{array}{c}\text { All Attribute } \\
\text { Visualization Time } \\
\text { (ms) }\end{array}$ & $\begin{array}{c}\text { All Default Attribute } \\
\text { Visualization Time } \\
\text { (ms) }\end{array}$ \\
\hline 20 & 855 & 356 \\
30 & 1101 & 506 \\
50 & 1682 & 841 \\
100 & 3504 & 1585 \\
300 & 9731 & 4386 \\
500 & 16,473 & 7263 \\
1000 & 32,179 & 14,002 \\
\hline
\end{tabular}

${ }^{a}$ Default attributes for Transcript feature are source_id, source_name, symbol and taxonomy_id. more than 1 undo/error). The perceived time, evaluated from 1 to 5 , got a mean of 3 , demonstrating the intuitiveness of the interface despite of the complexity of some tasks. Overall, all users showed satisfaction for their user experience. Observing them to perform the tasks pointed out some little usability issues, mainly about navigation of query composition Web pages and results visualization that will be tackled in future work.

\section{REFERENCES}

[1] M. Y. Galperin and X. M. Fernández-Suárez, “The 2012 Nucleic Acids Research Database Issue and the Online Molecular Biology Database Collection,” Nucleic Acids Research, Vol. 40, Database Issue, 2012, pp. D1-D8. http://dx.doi.org/10.1093/nar/gkr1196

[2] N. W. Paton, S. A. Khan, A. Hayes, F. Moussouni, A. Brass, K. Eilbeck, C. A. Goble, S. J. Hubbard and S. G. Oliver, "Conceptual Modeling of Genomic Information," Bioinformatics, Vol. 16, No. 6, 2000, pp. 548-557. http://dx.doi.org/10.1093/bioinformatics/16.6.548

[3] E. Bornberg-Bauer and N. W. Paton, "Conceptual Data Modelling for Bioinformatics," Briefings in Bioinformatics, Vol. 3, No. 2, 2002, pp. 166-180. http://dx.doi.org/10.1093/bib/3.2.166

[4] M. Masseroli, D. Martucci and F. Pinciroli, "GFINDer: Genome Function INtegrated Discoverer through Dynamic Annotation, Statistical Analysis, and Mining," Nucleic Acids Research, Vol. 32, 2004, pp. W293-W300. http://dx.doi.org/10.1093/nar/gkh432

[5] M. Masseroli, O. Galati and F. Pinciroli, "GFINDer: Genetic Disease and Phenotype Location Statistical Analysis and Mining of Dynamically Annotated Gene Lists," Nucleic Acids Research, Vol. 33, 2005, pp. W717-W723. http://dx.doi.org/10.1093/nar/gki454

[6] A. Canakoglu, G. Ghisalberti and M. Masseroli "Integration of Biomolecular Interaction Data in a Genomic and Proteomic Data Warehouse to Support Biomedical Knowledge Discovery,” In: E. Biganzoli, A. Vellido, F. Ambrogi and R. Tagliaferri, Eds., Computational Intelligence Methods for Bioinformatics and Biostatistics, Springer, Heidelberg, 2012, pp. 112-126. http://dx.doi.org/10.1007/978-3-642-35686-5_10

[7] J. Nielsen, “Usability Engineering,” Morgan Kaufmann, San Francisco, 1993. 